\title{
Measuring the Utility/Path Diversity Trade off in Multipath Protocols
}

\author{
Fern Y. Hunt \\ National Institute of Standards and Technology \\ Mail Stop 8910 \\ Gaithersburg, Maryland 20899 \\ fern.hunt@nist.gov
}

\author{
Vladimir Marbukh \\ National Institute of Standards and Technology \\ Mail Stop 8910 \\ Gaithersburg, Maryland 20899 \\ vladimir.marbukh@nist.gov
}

\begin{abstract}
We derive a model of congestion control where the trade-off between utility and path diversity can be investigated. In a network where there can be multiple routes between locations, each source $s$ is assigned a route according to an allocation scheme where the degree of randomness and therefore path diversity is controlled by $h_{s}$, the entropy of the distribution defined by the allocation. Model equations are derived from a network utility maximization problem and the results of the analysis of two networks with a single source destination are presented. We conclude for each such network there is a critical value of $h_{s}$ for which stable equilibrium solutions of the model equations exist and using the results of [9] it can be shown that they are also solutions of the original optimization problem. Treating $h_{s}$ as a parameter, the trade-off is discussed in terms of the behavior of the time averaged utility as a function of $h_{s}$.
\end{abstract}

\section{Categories and Subject Descriptors}

C.2 [Network Protocols]: [Routing Protocols]

Keywords:TCP/IP protocols, multipath routing, discrete dynamics,

\section{INTRODUCTION}

In recent years, network protocols have been interpreted as algorithms that solve a convex optimization problem ( see e.g. [13], [8]). In this set-up overall congestion control is formulated as a network utility maximization problem (NUM) that is solved in a distributed fashion by the various network layers. The network topology and the capacity of its links introduces constraints on the optimal solution. Then algorithms solve the problem by computing and modifying the primal and dual variables based on efficient communication between users, link and router layers of the network.

Permission to make digital or hard copies of all or part of this work for personal or classroom use is granted without fee provided that copies are not made or distributed for profit or commercial advantage and that copies bear this notice and the full citation on the first page. To copy otherwise, to republish, to post on servers or to redistribute to lists, requires prior specific permission and/or a fee. VALUETOOLS 2009, October 20-22, Pisa, Italy

Copyright (c) 2009 ICST 978-963-9799-70-7

DOI 10.4108/ICST.VALUETOOLS2009.7591
Since the work of Kelly et al [8] congestion control protocols have been seen as regulating user network transmission rates so that the objective function that is, the aggregate utility, is maximized subject to capacity constraints ([9], [3]). The utility function incorporates efficient utilization and fair allocation of resources among users. Significantly such functions have been identified for existing protocols such as TCP and BGP (Border Gateway Protocols) through a process of "reverse engineering", thus opening up opportunities for analysis and improvement of existing protocols as well as the development of new ones.

The paradigm just described has been extended to the problem of characterizing protocols that jointly control congestion and routing $([13],[3],[4],[6],[7])$. Aside from the obvious improvement in the utilization of network resources that could be gained by such an approach, there are benefits in this time of cybersecurity concerns to building adequate network robustness against route disruptions. However implicit in such a protocol is a tradeoff between robustness through path diversity on the one hand and network performance or utility on the other. Single path routing based on e.g. the OSPF (Open Shortest Path First) protocol can lead to route flapping instability and reduced utility. However splitting traffic equally across all paths regardless of cost would also imply reduced utility. The best tradeoff if it exists would have to navigate between these extremes. Thus the design of stable, implementable algorithms that achieve maximum aggregate utility remains a challenge.

Our purpose is to propose a generalized NUM optimization problem for joint congestion control routing where we can examine the behavior of the time averaged utility as a function of entropy, a model parameter that can be interpreted as a measure of path diversity. This paper is not presenting an algorithm to be implemented on a real network but is rather, a theoretical study of issues that must be addressed by any algorithm that solves the NUM problem we discuss. Our model contains a routing scheme that allocates users according to a fixed vector whose components sum to one. The degree of path diversity of the route or path allocation for a class of network users $s$, can be measured in terms of the entropy $h_{s}$ of the probability distribution defined by the allocation. We discuss two example networks with a single source and destination.

Treating $h_{s}$ as a parameter, we find that as we increase $h_{s}$ from 0 , the model equations fail to converge to an equilibrium until the entropy reaches a critical value (see Section 4 for TwoLinks and Section 5 for Diamond). Stable equi- 
libria exist for values of $h_{s}$ greater or equal to the critical value and we show that the corresponding equilibrium pair $\left(x_{s}^{*}, \beta_{s}^{*}\right)$ are solutions of the optimization problem.

We also calculate the time averaged aggregate utility for a range of values $h_{s}$ for each topology (see Figure 3, Figure 5). These graphs show how the utility decreases as the entropy increases and they illustrate the fact that one can always increase the utility for a route allocation that has entropy greater than the critical value by changing the allocation to one with a smaller entropy. This automatically decreases the mean route cost. A protocol based on the model we presented does not allow one to decrease the entropy below the critical value. We conjecture that the behavior of the model for entropy values less than the critical value corresponds to the unstable behavior seen in the single path TCP/IP protocols discussed in reference [13].

We now turn to a description of the organization of the paper. The NUM optimization problem for a general network is presented in the next section, is followed by a description of the model of the dynamics of the protocol in time. This can be found in Section 3. The relationship between the NUM problem, the dual optimization problem and the resulting model equations is not the main focus of this paper so we defer discussion of these points until the end of the paper in the appendix. There following the work in references [9], [13] it is shown that the model equations (6)-(9) implement a solution of the NUM optimization problem stated in equations (1)-(4). They are specialized to the TwoLinks topology (see equations in (10)) and the Diamond topology (equations (15)-(19)). A discussion of how the aggregate utility varies as a function of the parameter $h_{s}$ for these two topologies can be found in Section 4.1 and 5.1 respectively and finally the conclusion can be found in Section 6 .

\section{OPTIMIZATION PROBLEM}

Consider a planar network to be a graph with nodes representing physical nodes in the network and edges representing links. The link capacities are defined by the vector $\mathbf{c}=\left(c_{1}, c_{2}, \cdots, c_{L}\right)$. A user who requires bandwidth to transmit from one node to another in the network (or a single TCP session between those two nodes) is indexed by $s$, the index of the source-destination pair. For each $s$ the network operator assigns a bandwidth rate $x_{s}$ and uses a twice differentiable strictly concave utility function $U_{s}:\left[m_{s}, M_{s}\right] \rightarrow \mathbb{R}$. Here $m_{s}$ and $M_{s}$ are lower and upper bounds respectively on the bandwidth rate. $U_{s}\left(x_{s}\right)$ measures the degree of user satisfaction, network fairness and efficiency for the part of the network defined by $s$. Users in a source-destination class $s$ are assigned a path by edge routers so that the fraction of users allocated to path or route $r$ is $\beta_{s r}$ with $0<\beta_{s r} \leq 1$. We can regard $\beta_{s}=\left\{\beta_{s r}\right\}_{r \in \mathcal{R}_{s}}$ as a probability distribution because the fractions sum to 1 . Here $\mathcal{R}_{s}$ is the set of all paths available to $s$. The distribution is constrained so that the traffic on any link does not exceed the link capacity.

The optimization problem that our protocol seeks to solve is therefore:

$$
\begin{aligned}
& \max _{\beta \geq 0, \mathbf{x} \in X \mathbf{x} \geq 0} \sum_{s} U_{s}\left(x_{s}\right) \\
& \sum_{s} \sum_{r \in R_{s}(l)} \beta_{s r} x_{s} \leq c_{l} \\
& \forall s \sum_{r \in \mathcal{R}_{s}} \beta_{s r}=1, \quad \beta_{s r} \geq 0 \\
& -\sum_{r \in \mathcal{R}_{s}} \beta_{s r} \log \beta_{s r} \geq h_{s}
\end{aligned}
$$

$\mathbf{x}=\left\{x_{s}: s=1,2, \cdots S\right\}$ is the vector of source rates with each $x_{s} \in\left[m_{s}, M_{s}\right]$ where $m_{s} \geq 0$ and $R_{s}(l)$ is the set of routes used by source $s$ that require link $l$. In this paper we will take $m_{s}=0$ for all $s$ and $U_{s}\left(x_{s}\right)=w_{s}\left(1-\alpha_{s}\right) x_{s}^{1-\alpha_{s}}$ with $\alpha_{s}=2$. The matrix $\beta=\left\{\beta_{s r}\right\}_{s, r}$ is a set of probability distributions that define path allocations for each source $s$. The constraints in (2) state that all routes that use link $l$ i.e. routes $r$ in $R_{s}(l)$ of source $s$ be assigned bandwidth rates $\beta_{s r} x_{s}$ so that the total link load does not exceed the capacity $c_{l}$. Equation (3) is the usual requirement for probability distributions and equation (4) places a lower bound on the degree of randomness for the distribution $\left\{\beta_{s r}\right\}$ for source $s$. Indeed recall that for any allocation $\beta_{\mathbf{s}}$, the entropy of the associated probability distribution is:

$$
\mathrm{H}\left(\beta_{\mathbf{s}}\right)=-\left(\sum_{r \in \mathcal{R}_{s}} \beta_{s r} \log \beta_{s r}\right) .
$$

Thus equation (4) is just $\mathrm{H}\left(\beta_{\mathbf{s}}\right) \geq h_{s}$. By putting a lower bound on the entropy we exclude allocations that place a majority of traffic on too few paths. The remaining path allocations have a greater degree of diversity, insuring some robustness in the system depending on the values of $h_{s}$. Small values of $h_{s}$ indicate that the problem is closer to the single path case since allocations that use fewer paths are admissible but as $h_{s}$ increases we are closer to the equiprobable case (a version of the multi-path problem) and the resulting set of feasible allocations reduces to those that have a higher degree of path diversity and higher average path cost.

In [10] where the idea of random route allocation was first proposed, the authors introduced an adaptive algorithm involving non-constant values of $h_{s}$. Through simulations they demonstrated the trade-off between stability and utility. They found that the maximum utility occurs near the boundary between stability and instability.

\section{DESCRIPTION OF MODEL}

To describe the results of this paper, the model equations are presented next. As is customarily the case in a constrained optimization problem, dual variables- in our case the link costs, play an integral role in the solution of equations (1)-(4). The solution of the dual problem (if it exists) can be used to find the solution of NUM. To solve the dual problem, a projection gradient procedure is employed and as in previous works, (see references [9], [13]), the dynamics of the link costs $\left\{p_{l}, l=1,2, \cdots, L\right\}$ and route allocations at each iteration step is a model of the behavior of the protocol at each time step. Under appropriate conditions on $U_{s}$ and the initial link costs (see reference [5]) the iterations converge to an optimal cost vector $p^{*}$ corresponding to the optimal values of $\mathbf{x}^{*}$ and $\beta^{*}$ of equations (1)-(4). 


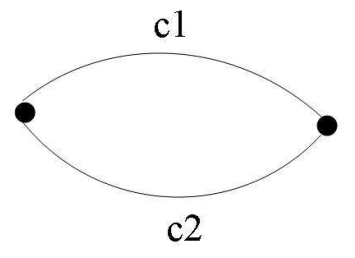

Figure 1:

If $p_{l}(k)$ is the link cost at time $k$ and $c_{l}$ is the capacity of the $l$ th link then the equations of the model are

$$
\begin{gathered}
p_{l}^{(k+1)}=\left[p_{l}^{(k)}-h\left\{c_{l}-\sum_{s} x_{s}(k) \sum_{r \in R_{s}(l)} \beta_{s r}^{(k)}\right\}\right]^{+} l=1, \cdots L \\
\beta_{s r}^{(k)}=\exp \left(-\gamma_{s}(k) d_{r}(k)\right) / Z_{s}(k)
\end{gathered}
$$

where $d_{r}(k)=\sum_{l \in r} p_{l}^{(k)}$ is the cost of route $r$ at time $k, h$ is a step size, $[a]^{+}=a$ if $a>0$ and is 0 otherwise.

$Z_{s}(k)=\sum_{r \in R(s)} \exp \left(-\gamma_{s}^{(k)} d_{r}(k)\right)$ is the normalization factor for the route distribution and, the variable $\gamma_{s}^{(k)}$ is the solution of the implicit equation,

$$
\gamma_{s}^{(k)} D_{s}(k)+\log \left(Z_{s}(k)\right)=h_{s}, \quad D_{s}=\sum_{r \in R(s)} \beta_{s r}^{(k)} d_{r}(k)
$$

The model equations are completed by a relation between the bandwidth rate $x_{s}(k)$ and $D_{s}(k)$, the mean route cost at time $k$ for positive constants $w_{s}$ and $M$.

$$
x_{s}(k)=\min \left(\left(\frac{w_{s}}{D_{s}(k)}\right)^{1 / 2}, M\right)
$$

Equations 7 and 8 force the route distribution $\beta_{s r}^{(k)}$ to be the unique distribution of entropy $h_{s}$ with the smallest mean route cost at each time step $k$. Thus the condition on the route distributions is $\mathrm{H}\left(\beta_{\mathbf{s}}\right)=h_{s}$. This constant entropy requirement will constrain the set of values $\left\{p_{l} \mid l=1 \cdots L\right\}$ for which a bounded $\gamma_{s}^{(k)}$ exists. The precise set depends on the network topology and capacity of the links. In the simple sample networks we discuss these regions can be determined.

\section{TWO LINKS TOPOLOGY}

A network consisting of a single source-destination pair of nodes connected by two links is depicted in Figure 1. The equations 6 become:

$$
\begin{aligned}
& p_{1}^{(k+1)}=\left[p_{1}^{(k)}-h\left\{c_{1}-\beta_{1}^{k} x_{s}(k)\right\}\right]^{+}, \\
& p_{2}^{(k+1)}=\left[p_{1}^{(k)}-h\left\{c_{2}-\beta_{2}^{k} x_{s}(k)\right\}\right]^{+}
\end{aligned}
$$

Each link is a path or route so $d_{i}=p_{i} \quad i=1,2$.

Assuming $c_{1}>c_{2}$, and initial link prices $p_{1}^{(0)}<p_{2}^{(0)}$, there is a critical entropy $h_{T}(c)<\log 2$ given by,

$$
h_{T}(c)=-\left[\frac{c_{1}}{c_{1}+c_{2}} \log \frac{c_{1}}{c_{1}+c_{2}}+\frac{c_{2}}{c_{1}+c_{2}} \log \frac{c_{2}}{c_{1}+c_{2}}\right]
$$

with a corresponding critical route distribution,

$$
\beta_{1}^{*}=\frac{c_{1}}{c_{1}+c_{2}}, \beta_{2}^{*}=\frac{c_{2}}{c_{1}+c_{2}}
$$

We say that $p_{i}^{(k)} \in W_{i}$ if $p_{i}^{k}-h\left\{c_{i}-\beta_{i}^{(k)} x s(k)\right\}>0$. Figure 2 shows the behavior of iterates of equations in 10. If $\mathcal{R}$ is the region bounded by the lines $L_{0}$, the $p_{2}$ axis, the line $L_{2}$ and the line $\left\{\mathbf{p} \mid p_{1}=p_{2}\right\}$, then $\mathcal{H}=\mathcal{R} \cap W_{1} \cap W_{2}$. Lines $L_{0}$ and $L_{2}$ are perpendicular to the line $\bar{x}^{*}=\beta_{1}^{*} p_{1}+\beta_{2}^{*} p_{2}$. If $\mathcal{J}=$ $\left\{\mathbf{p}\left|\mathbf{p}=\left(p_{1}, p_{2}\right)\right| p_{1}<p_{2}, \quad p_{1}>0, p_{2}>0\right\}$, then it can be shown ([5]) that iterates of a point $\mathbf{p}^{(\mathbf{0})}$ beginning in the region $\mathcal{H} \subset \mathcal{J}$ remain in $\mathcal{H}$ and converge to an equilibrium point on the line of points with constant average $p$ value $\bar{x}^{*}$ (see Figure 2). We claim in fact that if $p_{1}^{(k-1)}<p_{2}^{(k-1)}$, the allocations at time $k \geq 1$ satisfy, $\beta^{(k)}=\left(\beta_{1}^{(k)}, \beta_{2}^{(k)}\right)=$ $\beta^{*}=\left(\beta_{1}^{*}, \beta_{2}^{*}\right)$. To see this note that the implicit equation (8) forces the entropy of $\beta^{k)}$ to be $h_{T}(c)$. There are only are only two distributions with that entropy value and the conditions $p_{1}^{(k-1)}<p_{2}^{(k-1)}$ imply that $\beta_{1}^{(k)}>\beta_{2}^{(k)}$. Since $\beta^{*}$ is the unique distribution satisfying the latter condition the claim is proved. Each equilibrium point $\left(p_{1}^{*}, p_{2}^{*}\right)$ corresponds to the unique solution $\left(x s^{*}, \beta^{*}\right)$ of the optimization problem when $h$ is sufficiently small with,

$$
\begin{aligned}
x_{s}^{*} & =\left(\frac{w_{s}}{\bar{x}^{*}}\right)^{\frac{1}{2}} \\
{\overline{x_{s}}}^{*} & =\beta_{1}^{*} p_{1}^{*}+\beta_{2}^{*} p_{2}^{*}
\end{aligned}
$$

The proof of optimality follows along the same lines as the proof of Theorem 1 in [9] and uses [1] p. 214.

Orbits starting outside of $\mathcal{H}$ either converge to the intersection of the $p_{2}$ axis with the $\bar{x}^{*}$ line or they approach the line $p_{1}=p_{2}$ and fail to converge (see [5]).

In the case where $h_{s}<h_{T}(c)$ we can also conclude that the route allocation distribution is constant while the iterates are in the region $\mathcal{J}$. This is proved using the same reasoning as in the case $h_{s}=h_{T}(c)$. Here a point beginning in $\mathcal{J}$ will remain there and converge to a unique point $\left(0, p_{2}^{*}\right)$ on the $p_{2}$ axis. Thus the larger link 1 , is saturated, while link 2 reaches some positive equilibrium price. However the equilibrium bandwidth $x s^{*}$ will be smaller than the value achieved at $h_{T}(c)$. When $h_{s}<h_{T}(c)$, orbits leave $\mathcal{J}$ and move towards the line $\left\{\left(p_{1}, p_{2}\right): p_{1}=p_{2}\right\}$ as $k \rightarrow \infty$. Meanwhile, the solution of the implicit equation $\gamma^{k)} \rightarrow \infty$ and the corresponding entropy approaches $\log 2$. Thus the orbit leaves the feasible region and fails to converge. 


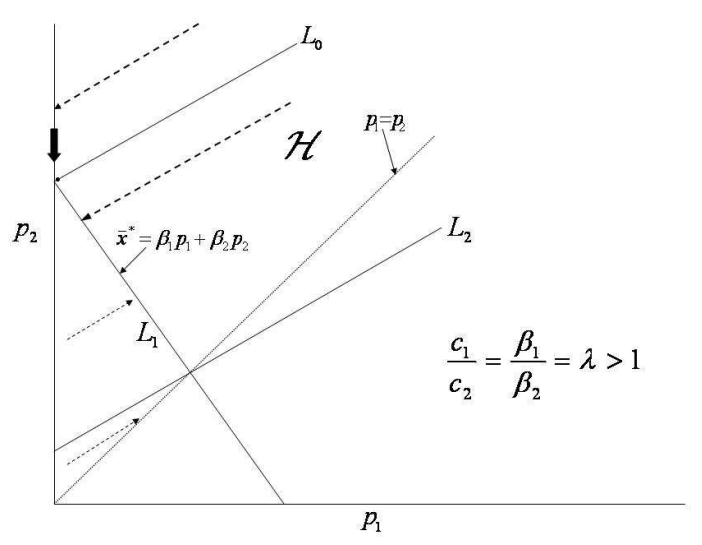

Figure 2: Iterates of Equation 10 (in brown and green) for $h_{s}=h_{T}(c)$

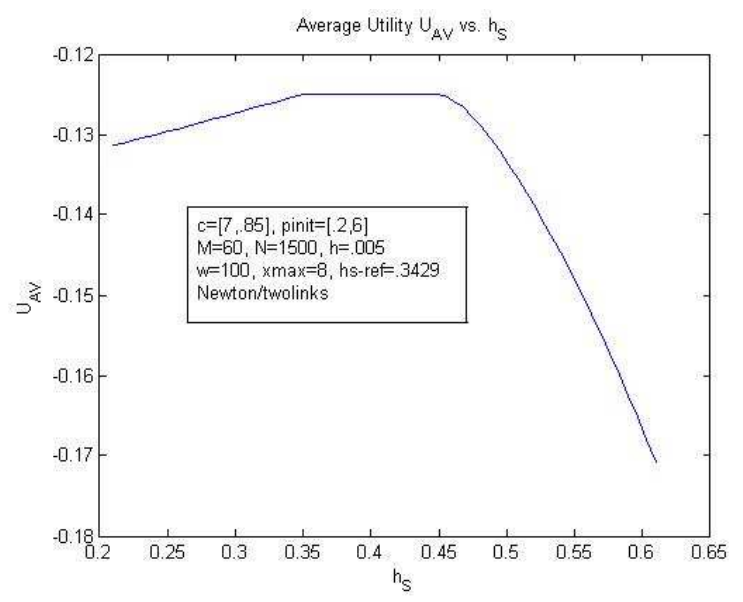

Figure 3: $h_{s}$-ref is the critical entropy $h_{T}(c)$.

\subsection{Utility/ Entropy tradeoff for TwoLinks}

For general multipath models the tradeoff between path diversity and utility is not well understood but introducing the entropy $h_{s}$ as a measure of path diversity, directly into our model equations allows us to address the question of tradeoff in terms of a study of the utility as a function of $h_{s}$. For the TwoLinks (and the Diamond) topology the utility was computed at each time step over a finite time interval. This was done for each $h_{s}$ over a range of values where the minimum $h_{s}$ was less than critical value $h_{T}(c)$ and the maximum $h_{s}$ was greater. An example of such a computation can be seen in Figure 3. As $h_{s}$ increases from 0 the average utility steadily increases and plateaus near the average utility value at $h_{T}(c)$ and then decreases for increasing $h_{s}>h_{T}(c)$. Thus (approximately) this graph mirrors the simulations by Marbukh and Klink [10] of a stochastic model where $h_{s}$ is adjusted adaptively. Here $N$ is the number of iterations performed at each $h_{s}$, and $M$ is the number of $h_{s}$ values.

\section{DIAMOND TOPOLOGY}

We turn our discussion now to another simple topology (see Figure 4 ) with 5 links attached to a single source destination pair. As seen in the figure there are 3 routes, using the links indicated in the figure.

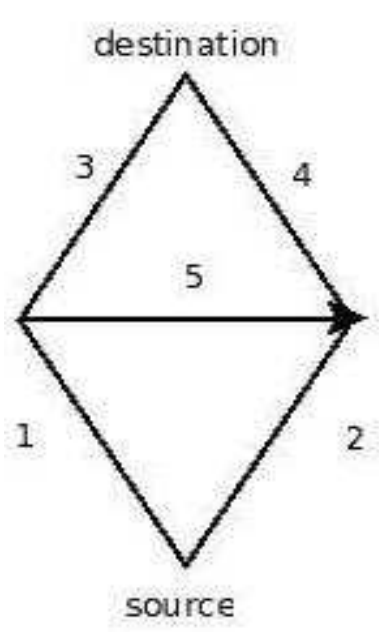

ROUTE \# LINKS

$\begin{array}{lr}1 & 1,3 \\ 2 & 2,4 \\ 3 & 1,5,4\end{array}$

Figure 4: DIAMOND NETWORK

Inspection of the figure leads to the system:

$$
\begin{aligned}
p_{1}^{(k+1)} & =\left[p_{1}^{(k)}-h\left\{c_{1}-\left(\beta_{1}^{(k)}+\beta_{3}^{(k)}\right) x_{s}(k)\right\}\right]^{+} \\
p_{2}^{(k+1)} & =\left[p_{2}^{(k)}-h\left\{c_{2}-\beta_{2}^{(k)} x_{s}(k)\right\}\right]^{+} \\
p_{3}^{(k+1)} & =\left[p_{3}^{(k)}-h\left\{c_{3}-\beta_{1}^{(k)} x_{s}(k)\right\}\right]^{+} \\
p_{4}^{(k+1)} & =\left[p_{4}^{(k)}-h\left\{c_{4}-\left(\beta_{2}^{(k)}+\beta_{3}^{(k)}\right) x_{s}(k)\right\}\right]^{+} \\
p_{5}^{(k+1)} & =\left[p_{5}^{(k)}-h\left\{c_{5}-\beta_{3}^{(k)} x_{s}(k)\right\}\right]^{+}
\end{aligned}
$$

where as in the previous section, the bandwidth rate is $x_{s}(k)=\min \left(\left(\frac{w_{s}}{\mathbb{E}\left[d^{(k)}\right]}\right)^{\frac{1}{2}}, M\right)$, with $\mathbb{E}\left[d^{(k)}\right]=\sum_{r=1}^{3} \beta_{r}^{k} d_{r}^{(k)}=$ $\sum_{r=1}^{3} \beta_{r}^{(k)}\left(\sum_{l^{\prime} \in r} p_{l^{\prime}}^{k}\right)$. The route probabilities are defined by the following equations:

$$
\beta_{i}^{(k)}=\frac{\exp \left(-\gamma^{(k)} d_{i}^{(k)}\right)}{Z(k)} \quad i=1,2,3
$$

where $Z(k)=\sum_{i=1}^{3} \exp \left(-\gamma^{(k)} d_{i}^{(k)}\right)$ and

$d_{1}^{(k)}=p_{1}^{(k)}+p_{3}^{(k)}, d_{2}^{(k)}=p_{2}^{(k)}+p_{4}^{(k)}, d_{3}^{(k)}=p_{1}^{(k)}+p_{5}^{(k)}+p_{4}^{(k)}$.

Here led by the search for strictly interior equilibria of equations (15)-(19) we take

$$
c_{2}=c_{3}, c_{1}=c_{3}+c_{5}, c_{4}=c_{2}+c_{5} .
$$

As motivation we can think of link 1 and 3 as an input/ output pair while links 2 and 4 are a corresponding input/output pair.

There are only two distributions $\beta=\left(\beta_{1}, \beta_{2}, \beta_{3}\right)$, with given entropy $h_{s}<\log 3$, satisfying the condition $\beta_{1}=$ 
$\beta_{2}$. The choices correspond to the inequalities, $\beta_{1}>\beta_{3}$ or $\beta_{1}<\beta_{3}$. Furthermore, if we choose initial link prices $p_{l}^{(0)} \quad l=1 \ldots 5$ that are compatible with equation (22) then $\beta_{1}^{(k)}=\beta_{2}^{(k)} \quad k \geq 1$.

Given $1 \leq i \leq 5$, for any $k \geq 0$ we will say that $p_{i}^{(k)} \in W_{i}$ if $p_{i}^{(k+1)}>0$.

Lemma 1. Suppose the capacities in equations (15)-(19) satisfy equation (22). Further suppose the initial conditions

$$
p_{2}^{(0)}=p_{3}^{(0)}, \quad p_{1}^{(0)}=p_{3}^{(0)}+p_{5}^{(0)}, \quad p_{4}^{(0)}=p_{2}^{(0)}+p_{5}^{(0)}
$$

hold, with $p_{i}^{(0)} \in W_{i}, i=1, \ldots 5$. Then

$$
p_{2}^{(k)}=p_{3}^{(k)}, \quad p_{1}^{(k)}=p_{4}^{(k)}, k \geq 1
$$

and $\beta_{1}^{(k)}=\beta_{2}^{(k)}$ for $k \geq 1$.

Proof. The proof is by induction on $k$.

The conclusion of the lemma thus implies that with appropriate choice of initial link prices there are at most two possible distributions $\beta$ with a given entropy $h_{s}$ and one can show that if $d_{3}^{(k)} \geq d_{1}^{(k)}=d_{2}^{(k)}$ then there is just one. Therefore as long as iterates remain in the region $\left\{\mathbf{p}: d_{1}<d_{3}\right\}$, $\left(\beta_{1}^{(k)}, \beta_{2}^{(k)}, \beta_{3}^{(k)}\right)$ is constant after one time step. In reference [5] we describe the analytical and computational study of equations (15)-(19) and find that the algorithm converges for values $h_{s} \geq h_{D}(c)$ where $h_{D}(c)$ is

$$
\begin{aligned}
h_{D}(c)=-[ & \frac{c_{2}}{c_{2}+c_{3}+c_{5}} \log \frac{c_{2}}{c_{2}+c_{3}+c_{5}}+ \\
& \frac{c_{3}}{c_{2}+c_{3}+c_{5}} \log \frac{c_{3}}{c_{2}+c_{3}+c_{5}}+ \\
& \left.\frac{c_{5}}{c_{2}+c_{3}+c_{5}} \log \frac{c_{5}}{c_{2}+c_{3}+c_{5}}\right]
\end{aligned}
$$

Using arguments similar to those used for TwoLinks at the critical value $h_{T}(c)$, we can show at $h_{s}=h_{D}(c)$ there exist a family of equilibria in the $d_{1}, d_{3}$ plane that lie along the line $\left\{\left(d_{1}, d_{2}, d_{3}\right): d_{1}=d_{2} \quad \bar{x}^{*}=\beta_{1}^{*} d_{1}+\beta_{2}^{*} d_{2}+\beta_{3}^{*} d_{3}\right\}$. These points correspond to a unique $\left(x_{s}^{*}, \beta^{*}\right)$ solution of the optimization problem where $\beta^{*}$ is the route distribution corresponding to the critical entropy $h_{D}(c)$,

$$
\beta^{*}=\left(\frac{c_{2}}{c_{2}+c_{3}+c_{5}}, \frac{c_{3}}{c_{2}+c_{3}+c_{5}}, \frac{c_{5}}{c_{2}+c_{3}+c_{5}}\right)
$$

The proof of optimality as in the TwoLinks case uses the fact that iterates stay within a region where the route distribution is constant.

Values of $h_{s}>h_{D}(c)$ corresponding to route distributions

$$
\beta=\left(\frac{\nu c_{2}}{c_{2}+c_{3}+c_{5}}, \frac{\nu c_{3}}{c_{2}+c_{3}+c_{5}}, \frac{c_{5}+(1-\nu)\left(c_{2}+c_{3}\right)}{c_{2}+c_{3}+c_{5}}\right)
$$

for $\nu<1$ were analyzed and computations were done. We show that under the hypotheses of Lemma 1 iterates of the algorithm converge to a unique equilibrium where $p_{i}^{*}=01 \leq$ $i \leq 4$, and $p_{5}^{*}>0$ when $h_{s}$ is the entropy of the distribution in equation 24. The algorithm fails to converge if $\nu>1$, a situation where $h_{s}<h_{D}(c)$. Here orbits approach the line in the $\left(d_{1}, d_{3}\right)$ plane $d_{1}=d_{3}$ as $k \rightarrow \infty$. Since we have $d_{1}^{(k)}=d_{2}^{(k)}$ for all $k \geq 1$ by Lemma 1 , the iterates

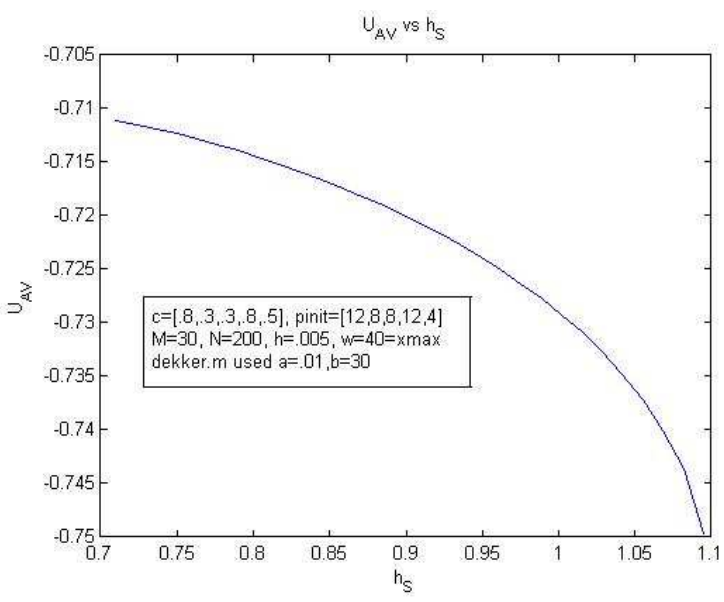

Figure 5: DIAMOND Utility vs. entropy

leave the region of feasibility. Thus we see that $h_{D}(c)$ marks the boundary between convergence and divergence of this algorithm.

\subsection{Utility/Entropy tradeoff for Diamond topol- ogy}

The variation of utility as a function of $h_{s}$ in the Diamond topology is illustrated in Figure 5. The average utility over a finite time interval (defined by the number of iterations $N$ ) was computed for a given value of $h_{s}$. Again the minimum $h_{s}$ was less than $h_{D}(c)$ while the maximum value was greater.

Although the average utility decreases for $h_{s} \geq h_{D}(c)$, the maximum is not achieved at the critical value. It continues to increase until the value $\log 2$ is reached. A plot of $\beta_{3}$, the fraction of traffic allocated to route 3 as a function of $h_{s}$ shows that $\beta_{3}$ decreases as $h_{s}$ decreases until $\beta_{3}=0$ at $\log 2$. We find this to be the case even when link 5 is not an obvious bottleneck e.g. when $c_{5}>c_{2}=c_{3}$. Thus the route with the cross link 5 is eliminated and the network is reduced effectively to TwoLinks. Numerically we find that we cannot even iterate long enough to calculate the average utility when $h_{s}<\log 2$.

\section{CONCLUSION}

We presented a network utility maximization problem that is equivalent to a convex optimization problem that incorporates a measure of path diversity in the assignment of paths to users transmitting between two locations in the network. Within the framework of our model it is possible to examine the tradeoffs between utility maximization that could be offered by single path routing on the one hand and robustness on the other, by introducing an entropy parameter $h_{s}$ directly into the optimization problem (and thus the model equations). One can then study the utility as a function of $h_{s}$. In this paper, the proposed method is demonstrated for two example topologies TwoLinks and Diamond. Thinking of $h_{s}=0$ as representing a single path scheme while $h_{s}=\log$ (number of routes) represents the most robust multipath scheme, we see that our results suggest that the degree to which a network can approach single path routing is limited by the link capacities and its topology. In each case, there is a critical value $h_{s}, h_{T}(c)$ in the case of TwoLinks and $h_{D}(c)$ for Diamond, so that model equations 
diverged for $h_{s}$ less than the critical value. On the other hand we presented sufficient conditions for the model equations to converge to equilibria that correspond to solutions of the optimization problem. The extension of this methodology to more complex networks is needed as well as the consideration of other approaches e.g. subgradient algorithms. It is possible that the problem of divergence could be addressed in this way.

\section{APPENDIX}

\section{A. DERIVATION OF THE MODEL}

The NUM problem in equations (1)-(4) is not convex in $(x, \beta)$ but through a change a variables $(x, \beta) \mapsto(x, y)$ it can be transformed to one. The new problem is:

$$
\begin{gathered}
\max _{x, y} \sum_{s} U_{s}\left(x_{s}\right) \\
\sum_{s} \sum_{r \in R_{s}(l)} y_{s r} \leq c_{l} \\
x_{s}=\sum_{r \in R_{s}} y_{s r}, \quad y_{s r} \geq 0 \\
-\sum_{r \in R_{s}} \frac{y_{s r}}{x_{s}} \log \frac{y_{s r}}{x_{s}} \geq h_{s}
\end{gathered}
$$

This is a convex program because the functions in equations ( 25)-(28) are clearly concave. If we assume the conditions

$$
\sum_{s^{\prime}} m_{s^{\prime}}<c_{l}
$$

hold for each $l$, where the summation is over all sourcedestination pairs $s^{\prime}$ that use link $l$, then there is a Slater point $([12])$ and the Slater constraint qualification is satisfied. Thus the problem in equations ( 25)-(28) is superconsistent and Lagrange multipliers exist. The Lagrangian for the problem is:

$$
\begin{gathered}
\mathfrak{L}(x, y, \lambda, p)=\sum_{s}\left[U_{s}\left(x_{s}\right)-\sum_{r \in R_{s}}\left(\sum_{l \in r} p_{l}\right) y_{s r}\right. \\
\left.-\lambda_{s}^{1}\left(\sum_{r \in R_{s}} y_{s r} \log \left(\frac{y_{s r}}{x_{s}}\right)+h_{s} x_{s}\right)-\lambda_{s}^{2}\left(x_{s}-\sum_{r \in R_{s}} y_{s r}\right)\right]+\sum_{l=1}^{L} p_{l} c_{l}
\end{gathered}
$$

where $\lambda=\left[\lambda_{s}^{1}, \lambda_{s}^{2}: s=1, \cdots S\right]$ are Lagrange multipliers for constraints in equations (27) and (28). To obtain solutions $x, y$ we consider the solutions of the problem;

$$
\max _{x, y} \mathfrak{L}(x, y, \lambda, p)=\max _{x} \max _{y} \mathfrak{L}(x, y, \lambda, p)
$$

where maximization is first performed in $y$ with $x$ and $p$ held fixed. The problem $\max _{y} \mathfrak{L}(x, y, \lambda, p)$ is equivalent to the constrained problem

$$
\begin{array}{r}
\max _{y} \sum_{s} U_{s}\left(x_{s}\right)-\sum_{r \in R_{s}}\left(\sum_{l \in r} p_{l}\right) y_{s r} \\
x_{s}=\sum_{r \in R_{s}} y_{s r}, \quad y_{s r} \geq 0 \\
-\sum_{r \in R_{s}} \frac{y_{s r}}{x_{s}} \log \left(\frac{y_{s r}}{x_{s}}\right) \geq h_{s}
\end{array}
$$

Problem equation (29) has a Slater point so it too is superconsistent. A solution $y^{*}$ to (29) exists if and only if there is a $\lambda^{*}$ that satisfies the KKT conditions ([12] p.182,183). Reintroducing $\beta_{s r}=\frac{y_{s r}}{x_{s}}$ where $\beta_{s r}$ is independent of $x_{s}$, we obtain the following form of the optimal solution of (29) for each fixed $x_{s}$ and $p$.

$$
\beta_{s r}=\frac{\exp \left(-\gamma * d_{r}\right)}{Z_{s}}
$$

where

$$
Z_{s}=\sum_{r \in R_{s}} \exp \left(-\gamma * d_{r}\right), \quad d_{r}=\sum_{l \in r} p_{l}
$$

and $\gamma=\frac{1}{\lambda_{s}^{2}}$ is the solution of the implicit equation,

$$
\gamma * D_{s}+\log Z_{s}(\gamma)=h_{s} \quad D_{s}=\sum_{r \in R_{s}} \beta_{s r} d_{r}
$$

Recognizing that equations (29) is equivalent to the problem of minimizing $\sum_{r \in R_{s}} y_{s r} d_{r}=x_{s} D_{s}$ subject to the constraints, we see that the optimal solution can be obtained by setting $\gamma$ equal to the unique positive root of equation (31). Indeed there are in general two roots of this equation when $h_{s}<\log \left|R_{s}\right|$ and the positive root provides the smallest value of $D_{s}$. Given $\gamma>0$, call any $\beta_{s r}$ subject to $(30)$ and $(31), \beta_{s r}^{*}$. Then we have $y_{s r}^{*}=x_{s} \beta_{s r}^{*}$. The $\left\{y_{s r}^{*}: r=1, \cdots R_{s}\right\}$ are unique since for each $s,(31)$ has a unique positive solution. Set

$$
L(x, p)=\max _{y} \mathfrak{L}(x, y, \lambda, p)=\mathfrak{L}\left(x, y^{*}(x, p), \lambda^{*}(x, p), p\right)
$$

where $y^{*}(x, p)$ and $\lambda^{*}(x, p)$ are the optimal solution and Lagrange multipliers of equation (29) for fixed $x$ and $p$. It can be shown that $L$ is convex in $p$. To see this note that

$$
-\Lambda_{s}(p)=-\sum_{r \in \mathcal{R}_{s}}\left(\sum_{l \in r} p_{l}\right) \beta_{s r}^{*} \geq-\sum_{r \in \mathcal{R}_{s}}\left(\sum_{l \in r} p_{l}\right) \beta_{r}
$$

and is the supremum over all vectors $\beta ; \beta: \sum_{r \in R_{s}} \beta_{r}=$ $1,-\sum_{r \in R_{s}} \beta_{r} \log \beta_{r} \geq h_{s}$. Thus $L(x, p)$ is the supremum of set of convex functions over an infinite (convex) set (p. $81[2]$ ). Applying the same reasoning to $L$ we can conclude that the dual function

$$
\mathcal{Q}(p)=\max _{x} L(x, p)=\max _{x} \max _{y} \mathfrak{L}(x, y, \lambda, p)
$$

is convex in $p$. The remainder of this derivation follows the work in references [9] and [13]. First the dual problem for $p$ is formulated as:

$$
\min _{p: p_{l} \geq 0} \mathcal{Q}(p)
$$

If $\Lambda$ is differentiable at $p$, Danskin's theorem [1] applied to $\mathfrak{L}$ implies the differentiability of $\mathcal{Q}$. Indeed the unique maximizing $\bar{x}$ and $y=x \hat{\beta}^{*}$ are differentiable in $p$ and $\lambda$.This can be seen in the case of $\hat{\beta}$ by using the necessary optimality condition $\frac{\partial \mathfrak{L}}{\partial \beta_{s r}}=0$. At $\bar{x}$, either $\overline{x_{s}}(p)=M_{s}$ or $\left.U_{s}^{\prime}\left(\overline{x_{s}}\right)(p)\right)=\Lambda_{s}(p)$. If $\overline{x_{s}}(p)<M_{s}$, then a condition introduced in [9], $U^{\prime \prime}\left(x_{s}\right) \geq \delta_{s}>0$ for all $x_{s} \in\left(0, M_{s}\right.$ ], together with the differentiability of $\Lambda_{s}$ with respect to $p$ and $\lambda$, imply that $\bar{x}_{s}$ is differentiable and thus $\mathfrak{L}\left(\bar{x}, y^{*}, \lambda, p\right)$ is differentiable. The condition that $\Lambda$ be differentiable at $p$ holds we conjecture for values of $p$ that lie in a convex subset. The exact nature of the subset depends on the existence of a finite positive root of equation (31). We have determined the region for sample problems discussed in this paper. In general however we do not expect $\mathcal{Q}$ to be everywhere differentiable. However when it is differentiable our interest lies 
in understanding the dynamics of the following equation for solving the optimization problem in equation (34):

$$
\begin{gathered}
p_{l}^{(k+1)}=\left[p_{l}^{(k)}-h\left\{c_{l}-\sum_{s} x_{s}^{(k)} \sum_{r \in R_{s}(l)} \beta_{s r}^{(k)}\left(\sum_{l^{\prime} \in r} p_{l^{\prime}}^{(k)}\right)\right\}\right]_{(35)}^{+} \\
l=1 \cdots N L
\end{gathered}
$$

The equations (35) are supplemented by the relations coming from the optimality conditions.

$$
\begin{gathered}
\beta_{s r}^{(k)}=\frac{\exp \left(-\gamma_{s}^{(k)}\left(\sum_{l \in r} p_{l}\right)\right)}{Z\left(\gamma_{s}^{(k)}\right)} \\
x_{s}^{(k)}=\min \left(\left(\frac{w_{s}}{\mathbb{E}[d]}\right)^{\frac{1}{\alpha}}, M\right), \quad \mathbb{E}[d]=\sum_{r \in \mathcal{R}_{s}} \beta_{s r}^{(k)}\left(\sum_{l^{\prime} \in r} p_{l^{\prime}}^{(k)}\right)
\end{gathered}
$$

where $\gamma_{s}^{(k)}$ is the solution of equation (31) using $\beta_{s r}^{(k)}$ and $Z\left(\gamma_{s}^{(k)}\right)$ is defined analogously to $Z(s)$. Equation (31) effectively insures that at each time step $k$ the route distribution $\left\{\beta_{s r}^{(k)}: r \in \mathcal{R}_{s}\right\}$ has entropy $h_{s}$ for every $s$.

\section{B. REFERENCES}

[1] D.P. Bertsekas, J. N.Tsitsiklis "Parallel and Distributed Computation: Numerical Methods", Prentice Hall, Englewood Cliffs, N. J. 1989.

[2] S.Boyd, L. Vandenberghe "Convex Optimization", Cambridge University Press, Cambridge UK, 2004.

[3] M.Chiang, S.H. Low, A.R. Calderbank, J.C. DOYLE "Layering as optimization decomposition: A mathematical theory of network architectures", Proceedings of the IEEE, Vol. 95, no. 1, pp. 252-312, 2007.

[4] J. He, M. Bresler, M. Chiang, J. Rexford "Towards Robust Multi-Layer Traffic Engineering: Optimization of Congestion Control and Routing" IEEE Journal on Selected Areas in Communications, Vol. 25, No. 5, pp.868-880, 2007.

[5] F.Hunt, V. MARBukH "A Model of Joint Congestion Control Through Random Assignment of Paths", NIST Internal Report, 2009.

[6] F.P.Kelly, T. Voice "Stability of end-to-end algorithms for joint routing and rate control" Computer Communication Review, Vol. 35, No. 2, pp. 5-12, 2005.

[7] X. Lin, N. Shroff, R. Srikant "On the Connection-Level Stability of Congestion-Controlled Communication Networks", IEEE Trnasactions on Information Theory, Vol. 54, No. 5, 2008.

[8] F.P. Kelly, A.K.Maulloo, D.K.H. Tan "Rate control in communication networks: shadow prices, proportaion fairness and stability." Journal of the Operational Research Society, Vol. 49, pp. 237-252, 1998.

[9] S.H. Low, D. E. LApsley "Optimization Flow Control I: Basic Algorithm and Convergence" IEEE/ACM Transactions on Networking Vol. 7, No. 6, pp.861-874, 1999 available at http://netlab.caltech.edu/pub.

[10] V. Marbukh, S. KLINK "Decentralized Control of Large Scale Networks as a Game with Local Interactions:Cross Layer TCP/IP Optimization" ValueTools07.

[11] Y. Matsumoto "An Introduction to Morse Theory" Translations of Mathematical Monographs, Vol. 208, American Mathematical Society, 2002.

[12] A. Peressini, F.Sullivan, J.Uhl "The Mathematics of Nonlinear Programming", Springer Verlag, New York, N.Y. , 1988.

[13] J.WAng, L. Li, S.H. Low, J.C.Doyle "Cross-Layer Optimization in TCP/IP networks" IEEE Transactions on Networking, Vol. 13, No. 3, pp. 568-582, 2005. 